\title{
Neural Generation of Diverse Questions using Answer Focus, Contextual and Linguistic Features
}

\author{
Vrindavan Harrison and Marilyn Walker \\ University of California Santa Cruz \\ Santa Cruz, CA, US \\ \{vharriso, mawalker\}eucsc.edu
}

\begin{abstract}
Question Generation is the task of automatically creating questions from textual input. In this work we present a new Attentional Encoder-Decoder Recurrent Neural Network model for automatic question generation. Our model incorporates linguistic features and an additional sentence embedding to capture meaning at both sentence and word levels. The linguistic features are designed to capture information related to named entity recognition, word case, and entity coreference resolution. In addition our model uses a copying mechanism and a special answer signal that enables generation of numerous diverse questions on a given sentence. Our model achieves state of the art results of 19.98 Bleu_4 on a benchmark Question Generation dataset, outperforming all previously published results by a significant margin. A human evaluation also shows that the added features improve the quality of the generated questions.
\end{abstract}

\section{Introduction}

Question Generation (QG) is the task of automatically generating questions from textual input (Rus et al., 2010). There are a wide variety of question types and forms, e.g., short answer, open ended, multiple choice, and gap questions, each require a different approach to generate. One distinguishing aspect of a QG system is the type of questions that it produces. This paper focuses on the generation of factoid short answer questions, i.e., questions that can be answered by a single short phrase, usually appearing directly in the input text.

The work of a QG system typically consists of three conceptual subtasks: Target Selection, Ques- tion Representation Construction, and Question Realization. In Target Selection, important sentences and words within those sentences are identified. During Question Representation Construction, suitable question-type and syntactic form are determined based on the characteristics of the sentence at hand and the words it contains. An example of this can be seen in Agarwal et al. (2011) who define rules based on the discourse connectives in a sentence to decide which question-type is most appropriate. In the Question Realization step, the final surface form of the question is created.

It is common for QG systems to use a combination of semantic pattern matching, syntactic features, and template methods to create questions. Typically these systems look for patterns of syntax, keywords, or semantic roles that appear in the input sentence. Then they use these patterns to choose an appropriate question template, or use syntactic features to perform manipulations on the sentence to produce a question.

These rule-based systems have some strengths over Neural Network models: they are easier to interpret and allow developers greater control over model behavior. Furthermore, they typically require less data to develop than a complex Neural Network might need to achieve a similar level of performance. However, rule-based systems have some weaknesses as well. They tend to be laborious to develop, or domain specific. For example the system developed by Mostow and Chen (2009) relies on the presence of one of a set of 239 modal verbs in a sentence, and Olney et al. (2012) use 3000 keywords provided by the glossary of a Biology text book and a test-prep study guide. The system described in Chali and Hasan (2015) uses roughly 350 hand-crafted rules. Furthermore, these systems rely heavily on syntactic parsers, and may struggle to recover from parser inaccuracies. 


\begin{tabular}{l|l}
\hline$\#$ & \multicolumn{1}{c}{ Sentence } \\
S1 & $\begin{array}{l}\text { The character of midna has the most voice acting - her } \\
\text { on-screen dialog is often accompanied by a babble of } \\
\text { pseudo-speech, which was produced by scrambling the } \\
\text { phonemes of english phrases [ better source needed ] } \\
\text { sampled by japanese voice actress akiko komoto. }\end{array}$ \\
\hline Q1 & which person has the most spoken dialogue in the game? \\
\hline Q2 & who provided the basis for midna's voice? \\
\hline Q3 & what country does akiko komoto come from? \\
\hline Q4 & whaseline \\
\hline & \multicolumn{2}{|c}{ Our Model: FocusCR } \\
\hline Q5 & what character has the most voice acting in english? \\
\hline Q6 & what is the name of the japanese voice actress? \\
\hline Q7 & what is the nationality of akiko komoto? \\
\hline
\end{tabular}

Table 1: Sentence and associated questions generated from the baseline and our best model.

Among many different approaches to question generation, our work is most similar to recent work applying neural network models to the task of generating short answer factoid questions for SQUAD (Du et al., 2017; Yuan et al., 2017; Sachan and Xing, 2018). However these previous models have several limitations. As illustrated in Table 1, the SQUAD corpus (Rajpurkar et al., 2016) provides multiple gold standard references for each sentence (Q1, Q2, and Q3), but previous work to date can only generate one question for each sentence as represented by the baseline model (Q4), whereas our model can generate multiple questions as shown in Table 1.

In Section 2, we present our novel model that introduces additional token supervision representing features of the text as well as an additional lower dimensional word embedding. The features include a Named Entity Recognition (NER) feature, a word case feature, and a special answer signaling feature. The answer signaling feature allows our model to generate multiple questions for each sentence, illustrated with Q5, Q6 and Q7 in Table 1. We also introduce a coreference resolution model and supplement the sentence input representation with resolved coreferences, as well as a copying mechanism. Section 3 presents an evaluation of the final model on the benchmark SQuAD testset using automatic evaluation metrics and shows that it achieves state of the art results of 19.98 BLEU_4, 22.26 METEOR, and 48.23 ROUGE (Papineni et al., 2002; Banerjee and Lavie, 2005; Lin, 2004). To our knowledge this model outperforms all previously published results by a significant margin. A human evalu- ation also shows that the introduced features and answer-specific sentence embedding improve the quality of the generated questions. We delay a more detailed review of previous work to Section 4 and conclude in Section 5.

\section{Model}

Our QG model follows a standard RNN EncoderDecoder model (Sutskever et al., 2014) that maps a source sequence (declarative sentence) to a target sequence (question). The architecture of the baseline model is as follows: the encoder is a multi-layer bidirectional LSTM (Hochreiter and Schmidhuber, 1997) and the decoder is a unidirectional LSTM that uses global attention with input-feeding (Luong et al., 2015). This baseline model yields one question per sentence (Q4 in Table 1).

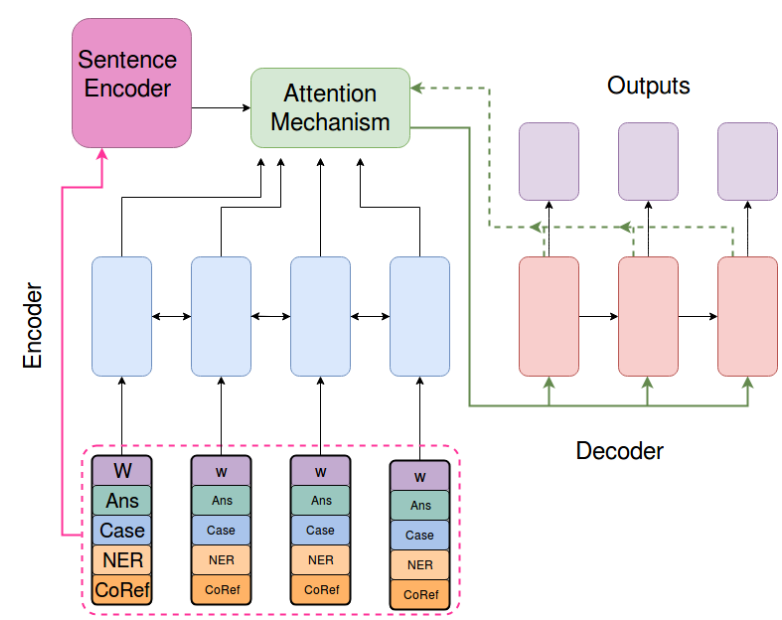

Figure 1: Diagram of our answer focus model.

We create our model by enhancing the baseline model in the following three ways:

- We add 4 different token level supervision features to the input. See Section 2.1.

- We add a sentence encoder that creates a question specific sentence embedding.

- We use a copy mechanism (See et al., 2017) to copy words from the sentence directly into the question.

\subsection{Feature Supervision}

A feature-rich encoding is constructed by concatenating several token level features onto the token's word-based embedding using a method similar to that described by Nallapati et al. (2016) for abstractive text summarization. 


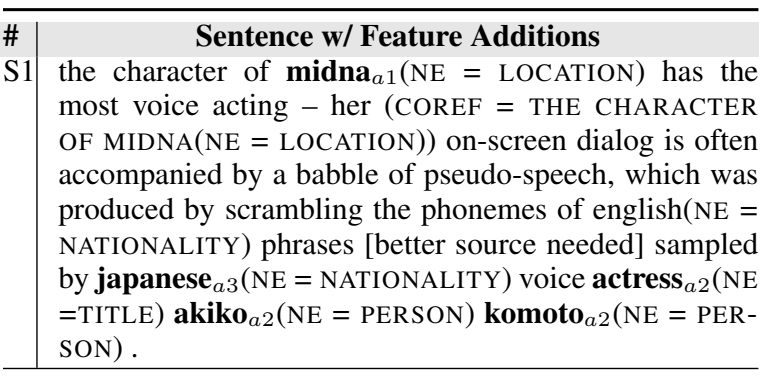

Table 2: Feature Markup on S1

Answer Signal Feature. It is usually the case that multiple questions can be asked about information contained within a single sentence. Therefore, a model that is capable of generating multiple questions for a single sentence has greater utility than a model such as the one described by Du et al. (2017) which is capable of generating only a single question per unique input sentence. This need to generate multiple questions for a sentence motivates our use of an answer signal. The model described by Yuan et al. (2017) also uses an answer signal feature. However, by combining it with additional features and the question specific sentence encoder our model achieves better results, as we show in Section 3.

The answer signal is equivalent to the output of the target selection module in a standard QG pipeline; this is provided as part of the SQUAD corpus, but is straightforward to calculate automatically.

The Answer Signal feature guides the model in deciding which information to focus on when reading the sentence. The signal being active in some location of the sentence indicates the answer to the question being generated. Then, modifying the location of the answer signal and keeping the rest of the sentence fixed enables the model to generate multiple answer specific questions per a given sentence.

The Answer Signal feature is implemented as a binary feature indicating whether or not a given token is part of the answer span. Table 2 illustrates the results of the answer signal feature on $\mathrm{S} 1$ from Table 1. The answer signals are shown in bold and annotated with an index. The indices $a 1, a 2$ and $a 3$ correspond to Q5, Q6 and Q7, respectfully, from Table 1 (as generated by our best model). For the sake of brevity we have simultaneously accentuated three separate answer signals in Table 2. In actuality, the model sees only one answer signal series per sentence input. To gener- ate questions Q5, Q6, and Q7 the model was fed the same sentence three separate times, each time with only one of $a 1, a 2$ or $a 3$ activated.

Case Feature. The case feature is a simple binary feature that represents whether or not the input token contains an upper case letter.

NER Feature. We use an NER feature that is designed in the same fashion as Nallapati et al. (2016), who have previously used an NER based feature embedding to improve performance of their sequence-to-sequence model used for Abstractive Text Summarization. Just as in Abstractive Text Summarization, identifying important entities that are central to the meaning of a sentence is an imperative component of the QG task.

The NER labels are computed in a preprocessing step. The result of NER labeling performed on a sentence is shown in Table 2. Similar to traditional word embeddings, we build a lookup based embedding for each NER label. During execution, the embedding associated with each token's NER label is retrieved using a table look-up and then concatenated onto the word embedding. The NER embeddings are a trainable parameter which get updated during the model's training process. Figure 1 shows a diagram depicting how the NER feature is incorporated into each token's feature rich encoding via concatenation.

Coreference Feature. Coreference labels are computed automatically in a pre-processing step. The coreference labels are calculated using all of the prior context for the input sentence text, but the input to the model is just the sentence augmented with the additional feature input. Table 2 shows how the NER and coreference features are expectedly noisy. Nevertheless, they improve the model as we show below.

Table 3 provides a detailed example for coreference showing context and the input sentence representation with and without the coreference feature, as well as the effect on the questions generated by the model. It is easy to see the benefits of the coreference representation qualititively on individual examples. For example, without coreference the model finds an entity elizabeth who is associated with net worth in the language model and uses that entity to generate the question rather than using beyoncé, the entity in context. In Section 3 we show that this qualitative difference affects the quantitative performance measures.

Coreference information is incorporated into 


\begin{tabular}{|c|c|}
\hline \multirow{2}{*}{$\begin{array}{c}\# \\
\mathrm{C} 1-33\end{array}$} & Partial Context \\
\hline & $\begin{array}{l}\text { In June } 2014 \text {, Beyoncé ranked at \#1 on the } \\
\text { Forbes Celebrity } 100 \text { list, earning an esti- } \\
\text { mated } \$ 115 \text { million throughout June } 2013 \\
\text { June } 2014 \text {. This in turn was the first time } \\
\text { she had topped the Celebrity } 100 \text { list as well } \\
\text { as being her highest yearly earnings to date. }\end{array}$ \\
\hline \multicolumn{2}{|r|}{ Sentence } \\
\hline $\mathrm{S} 2$ & $\begin{array}{l}\text { As of May } 2015 \text {, her net worth is estimated } \\
\text { to be } \$ 250 \text { million. }\end{array}$ \\
\hline \multicolumn{2}{|r|}{ Model Input with Coreference } \\
\hline $\mathrm{S} 2 \mathrm{w} / \mathrm{coref}$ & $\begin{array}{l}\text { As of may }(\mathrm{NE}=\mathrm{DATE}) 2015(\mathrm{NE}=\mathrm{DATE}) \text {, } \\
\text { her }(\mathrm{COREF}=\mathrm{BEYONCE}(\mathrm{NE}=\mathrm{PERSON}) \text { 's) } \\
\text { net worth is estimated to be } \$(\mathrm{NE}=\mathrm{MONEY}) \\
250(\mathrm{NE}=\text { MONEY }) \text { million(NE = MONEY }) \text {. } \\
\text { what is beyoncé's net worth in } 2015 ?\end{array}$ \\
\hline \multicolumn{2}{|r|}{ Model Input w/out Coreference } \\
\hline S2 no coref & $\begin{array}{l}\text { As of may }(\mathrm{NE}=\text { DATE }) 2015(\mathrm{NE}=\mathrm{DATE}) \text {, } \\
\text { her net worth is estimated to be } \$(\mathrm{NE}= \\
\text { MONEY }) 250(\mathrm{NE}=\text { MONEY }) \text { million(NE }= \\
\text { MONEY). } \\
\text { what is elizabeth's net worth in may } 2015 \text { ? }\end{array}$ \\
\hline
\end{tabular}

Table 3: Context, Sentence and Questions generated with and without coreference.

the model by augmenting the input text as shown in Table 3. The representative mention of each entity gets inserted into the sentence following its coreferent. This results in two phrases referencing the same entity appearing in the text one immediately following the other, the first being the one that appeared in the original text and the second being the entity's representative mention. Each token is assigned a binary feature indicating whether the token was in the original text or if it has been thus inserted. This way the model can learn to include or ignore the augmenting text as it deems necessary.

\subsection{Answer Focused Sentence Embedding}

The input sentence is encoded using a multi-layer bidirectional LSTM distinct from the token level encoder as illustrated in Figure 1. After completing the calculations of the last time step, the final state of the LSTM is taken as a sentence embedding. Then, this sentence embedding is concatenated on to the token level encoding for each time step. This allows the answer-specific sentence embedding to influence decoding decisions during each time step of the decoding process.

We experiment with pre-training the sentence encoder. The pre-training process is carried out in two steps. First, to facilitate training of the sentence encoder by itself, we need some ground truth sentence representation from which to measure similarity. Since this sentence encoder is used in question generation, it would be helpful to encode the sentence in such a way as to maximize its benefit to the QG model. With this as motivation, we train an instance of the full QG encoderdecoder model with the sentence encoder, but the sentence encoder is given the target question as input instead of the sentence. As expected, in this setting the model learns to generate questions very well because it "cheats" by taking the target as input. Next, the trained sentence encoder - that was trained by encoding the target questions - is decoupled from the full model for use in the following step.

In the second step, the question embeddings produced by the earlier trained encoder are used as ground truth representations from which to maximize similarity. Now, a new sentence encoder is trained that takes as input a declarative sentence. The new sentence encoder is trained to maximize the similarity between the input sentence's embedding and the question embedding produced by the earlier trained encoder - belonging to a specific question associated with the sentence. Specifically, loss is calculated between a sentence embedding $s$ and a ground truth representation $q$ using a Cosine Embedding Loss defined by:

$$
\operatorname{loss}(s, q)=1-\cos (s, q)
$$

where $\cos (s, q)$ is traditional cosine distance.

The pre-trained sentence encoder is then used to initialize the sentence encoder used in training a new instance of the full QG model.

\section{Experimental Setup and Results}

We conduct experiments exploring the effects of each of our model enhancements and train and evaluate all the models using the SQuAD dataset (Rajpurkar et al., 2016). We evaluate the models using both automatic evaluation metrics and a human evaluation using crowd sourced workers. In addition, we perform ablation tests to experiment with different feature settings. We name our two best models Focus and FocusCR, where FocusCR uses the coreference feature, and Focus does not.

Dataset. SQuAD is a dataset of over one hundred thousand (document, question, answer) tuples. The documents are Wikipedia articles and the questions are created by crowd workers. Answers to the questions are subsequently created by a separate group of crowd workers who select as the question's answer a span of text from 


\begin{tabular}{l|l}
\hline \# Model & \multicolumn{1}{|c}{ Sentences and Examples } \\
\hline S3 & $\begin{array}{l}\text { West got his big break in the year 2000, } \\
\text { when he began to produce for artists on } \\
\text { Roc-A-Fella Records. }\end{array}$ \\
\hline Q10 Copy & $\begin{array}{l}\text { when did west got his big break? } \\
\text { in what year did " big break " begin? }\end{array}$ \\
\hline \hline S4 & $\begin{array}{l}\text { A high-definition remaster of the game, } \\
\text { The Legend of Zelda: Twilight Princess } \\
\text { HD, is being developed by Tantalus Me- } \\
\text { dia for the Wii U. }\end{array}$ \\
\hline Q12 Copy & $\begin{array}{l}\text { who developed the legend of zelda? } \\
\text { who is the creator of the soundtrack of } \\
\text { mortal kombat? }\end{array}$ \\
\hline \hline S5 & $\begin{array}{l}\text { Both six- and seven-track versions of } \\
\text { the game's soundtrack were released on } \\
\text { November 19, 2006, as part of a Nintendo } \\
\text { Power promotion and bundled with repli- } \\
\text { cas of the Master Sword and the Hylian } \\
\text { Shield. }\end{array}$ \\
\hline \hline Q14 Copy & $\begin{array}{l}\text { What was released on november 19, 2006? } \\
\text { What was released on september 17, }\end{array}$ \\
\hline 2006?
\end{tabular}

Table 4: Question Generation with and without the Copy mechanism.

within the article. The creators of SQuAD keep part of the dataset private to be used as a hidden evaluation set in Question Answering tasks. For this work we use the roughly 92,000 examples that are publicly available. The 92,000 examples are partitioned into training (roughly 70k examples), development (roughly 10k examples), and test (roughly $11 \mathrm{k}$ examples) subsets. For the sake of comparison, we have used the same partitioning as Du et al. (2017) who have kindly made their data setting available on-line.

Using Stanford CoreNLP (Finkel et al., 2005; Manning et al., 2014) the data is tokenized, and NER and Coreference Resolution are performed. All the feature used by our model are calculated at this stage. Finally, the text is lowercased. We calculate separate source and target vocabularies of size 45,000 and 28,000, respectfully. Tokens that fall out of vocabulary (OOV) are represented with a special UNK token. In retrospect, separate vocabularies are not necessary for this task. We remove examples that have sentences or questions over 100 and 50 words long, respectfully.

Model Implementation. Our model is imple- mented using PyTorch ${ }^{1}$ and OpenNMT-py ${ }^{2}$ which is a PyTorch port of OpenNMT(Klein et al., 2017). The encoder, decoder, and sentence encoder are multi-layer RNNs, each with two layers. We use bi-directional LSTM cells with 640 units. The model is trained using Dropout (Srivastava et al., 2014) of 0.3 between RNN layers. Word embeddings are initialized using Glove 300 dimensional word vectors (Pennington et al., 2014) that are not updated during training. The sentence encoder is initialized using the pre-training process described in Section 2.2. All other model parameters are initialized using Glorot initialization (Glorot and Bengio, 2010).

The model parameters are optimized using Stochastic Gradient Descent with mini-batches of size 64. Beam search with five beams is used during inference and OOV words are replaced using the token of highest attention weight in the source sentence. We tune our model with the development dataset and select the model of lowest Perplexity to evaluate on the test dataset.

\subsection{Automatic Evaluation}

We compare our system's results to that of several other QG systems. The rows of Table 5 with labels $\mathrm{H} \& S$, Yuan, Du, and S\&X refer to the models presented in Heilman and Smith (2010a); Yuan et al. (2017); Du et al. (2017), and Sachan and Xing (2018), respectfully. Please refer to Section 4 Related Work for further details on each of these systems. The results of the H\&S system are reported in this work for the sake of comparison. The actual experiments were performed by Du et al. (2017) who describe the specific configuration of $H \& S$ in greater detail.

Results. We use BLEU score (Papineni et al., 2002) as an automatic evaluation metric and compare directly to other work. BLEU measures the similarity between a generated text called a candidate and a set of human written texts called a reference set. The score is calculated by comparing the n-grams of the candidate with the n-grams of the reference texts and then counting the number of matches.

Unfortunately there are inconsistencies in the method by which previous works have used BLEU to evaluate QG models. Therefore, to accurately compare BLEU scores, we evaluate our model us-

\footnotetext{
1 pytorch.org

${ }^{2}$ github.com/OpenNMT/OpenNMT-py
} 


\begin{tabular}{llll} 
Model & BLEU_4 & METEOR & ROUGE \\
\hline baseline & 11.53 & 15.93 & 39.57 \\
${\mathrm{H} \& \mathrm{~S}^{*}}^{*}$ & 11.18 & 15.95 & 30.98 \\
$\mathrm{Du}$ & 12.28 & 16.62 & 39.75 \\
$\mathrm{~S} \& \mathrm{X}$ & 14.37 & 18.57 & 42.73 \\
\hline FocusCR & 19.86 & 21.96 & $\mathbf{4 8 . 3 5}$ \\
Focus & $\mathbf{1 9 . 9 8}$ & $\mathbf{2 2 . 2 6}$ & 48.23
\end{tabular}

Table 5: System performance in automatic evaluation.

ing two different setups. First, when calculating BLEU for a given hypothesis question, some publications have used a reference set containing all the ground-truth questions corresponding to the sentence from which the hypothesis was generated. Table 5 shows our model's results compared to previous work using this setup of BLEU and the same partitioning of the SQuAD dataset.

Each of our models outperform previously published results in each of the BLEU, METEOR, and ROUGE categories by a significant margin. FocusCR is the second highest performing system and achieves an impressive BLEU_4 score of 19.86 , which greatly improves on the third highest BLEU_4 score of 14.37 belonging to $S \& X$. Focus gets a BLEU_4 score of 19.98 and is the best performing system overall.

In the second setup, for a given hypothesis question, Yuan et al. (2017) used a reference set containing only a single ground-truth question that corresponds to the same sentence and answer span from which the hypothesis was generated. We use this setup to evaluate our Focus and FocusCR models. The results are shown in Table 6. Here, Focus and FocusCR are the same models as shown in Table 5, with the only difference being the setting under which they are evaluated. Again, FocusCR achieves the second highest score and Focus gets the highest BLEU_4 score at 14.39. While the datasets in aggregate are the same, our partitioning of training, development, and test datasets is different from that of Yuan et al. (2017).

We perform ablation experiments to study the effects of each feature incorporated into the model. The results of these experiments can be seen in Table 8. With the exception of the CoRef feature, each feature added produces an improvement in BLEU, with the answer feature producing the greatest improvement. The copy-mechanism and sentence embedding, which is called Focus in the

\begin{tabular}{ll} 
Model & BLEU_4 \\
\hline baseline & 8.45 \\
Yuan & 10.5 \\
FocusCR & 14.16 \\
Focus & $\mathbf{1 4 . 3 9}$
\end{tabular}

Table 6: BLEU-4 scores when using answerspecific ground-truth questions as reference texts.

table, each increase performance further.

We also examine the effect of pre-training the sentence encoder as described in Section 2.2. In Table 8, the Focus and FocusCR models use a pre-trained sentence encoder. The sentence encoder used by FocusCR-npt is not pre-trained. We find that the pre-training has a positive effect on BLEU scores with FocusCR-npt getting BLEU 13.99, compared to the FocusCR getting BLEU 14.16.

Table 8 suggests that the coreference mechanism actually hurts performance as measured by BLEU but the example shown in Table 3 and the additional examples shown in Table 10 suggest that it is very effective.

Table 4 provides examples of the effect of the copy mechanism. Again, as with coreference, it is easy to see the benefits of the copying mechanism qualititively. For example, in Q14 and Q15 the model can effectively copy the right date into the question. In Q17, without copying, the model finds an entity von neumann who is associated with teaching and university in the model and uses that entity to generate the question rather than west the entity in context.

Question Diversity. We are interested in how the new features effect the quantity of unique questions produced by our model. Therefore, we counted the number of unique questions output by the model when considering the entire testing set as inputs. Here, we measure similarity using a strict character match comparison. Table 7 shows the results. We can see that the FocusCR model produces 10,194 unique questions, which is a $55 \%$

\begin{tabular}{lr} 
Model & Unique Q's \\
\hline Baseline & 6,595 \\
FocusCR & 10,194 \\
Human & 11,801
\end{tabular}

Table 7: Amount of unique questions generated. 


\begin{tabular}{llll} 
Model & BLEU_4 & METEOR & ROUGE \\
\hline baseline & 7.62 & 13.41 & 34.19 \\
+ Answer & 11.15 & 16.64 & 40.39 \\
+ NER & 11.54 & 16.94 & 40.93 \\
+ Case & 11.56 & 16.98 & 40.96 \\
+ CoRef & 10.28 & 16.14 & 39.22 \\
+ Copy & 13.00 & 18.43 & 42.78 \\
\hline FocusCR-npt & 13.99 & - & - \\
FocusCR & 14.16 & 19.24 & 43.07 \\
Focus & 14.39 & 19.54 & 43.00
\end{tabular}

Table 8: Results of ablation test.

increase over the 6,595 unique questions produced by the Baseline model. Although strict character matching is a crude method of measuring question similarity, we conclude that the features incorporated into the FocusCR model have a positive effect on the diversity of generated questions.

\subsection{Human Evaluation}

We perform human evaluation using crowd workers on Amazon Mechanical Turk ${ }^{3}$. The Turkers rate a pool of questions constructed by randomly selecting questions and their associated text passages from the test set. We select 114 questions each from the test dataset, the questions generated by the baseline model, and the questions generated by our FocusCR model. The questions are selected such that they all correspond to the same declarative sentence. In other words, we construct a set of 114 tuples where each tuple consists of one text passage, two model generated questions, and one human authored question.

We use a qualification criteria to restrict the participation of Turkers in our evaluation study. The Turkers must have above 95\% HIT approval rate with at least 500 HITs previously approved. Furthermore, Turkers are required to be located in English speaking countries. Turkers recieved $\$ 0.1$ for completing each HIT.

We closely follow the experiment design described by Heilman and Smith (2010b), who instruct Turkers to produce a single five-point quality rating per question. They provide Turkers with the following four reasons to downgrade a question: (Un)grammaticality, Incorrect Information, Vagueness, and Awkwardness. In our evaluation study, we use four categories of evaluation that resemble these criteria.

Turkers are asked to rate each question across

\footnotetext{
${ }^{3}$ www . mturk . com
}

four categories: Grammaticality, Correct Information, Answerability, and Naturalness. Grammaticality encompasses things like adherence to rules of syntax, use of the wrong $w h$-word, verb tense consistency, and overall legitimacy as an English sentence. The Correct Information category considers whether or not the question is related to the text passage (e.g., asking about Madonna when the passage is about Beyonce), implies something that is obviously incorrect, or contradicts information given in the text passage. The Answerability category reflects how much of the information required to correctly answer the question is contained within the text passage. Also, it considers whether or not the question has a clear answer, or is too vague (e.g., "What is it?"). The Naturalness category reflects how natural the question reads and considers whether or not it has some awkward phrasing. The Naturalness category also encompasses any other problems in the question that do not fall in the previous categories.

During evaluation, the Turker is presented with the text passage and its three corresponding questions in scrambled order. They are asked to give a rating from worst (1) to best (5) in each category for each question. Each HIT contains three text passages and a total of nine questions. Each HIT is assigned to three Turkers resulting in three ratings per question.

Results. Table 9 shows an average of the ratings assigned by the Turkers in each category. Answerability is the category in which the FocusCR model has the greatest improvement over the Baseline. In this category, FocusCR receives an average rating of 4.13, compared to the baseline's average rating of 3.73. FocusCR also outperforms the Baseline model in the Correct Information category with average ratings 4.13 and 3.78 , respectfully. In the Grammaticality and Naturalness categories the Baseline model has average ratings of 4.23 and 4.10 , respectfully. The FocusCR model has average ratings of 4.20 and 4.09 in the Grammaticality and Naturalness categories. The human authored questions outperform both models by a significant margin in all categories.

We note that there is only a slight difference between ratings achieved by the Baseline and FocusCR models in the Grammaticality and Naturalness categories. Yet, in both these categories the Baseline model slightly outperforms FocusCR. 
We suspect this is due to the brevity and generality of questions produced by the Baseline model. In contrast, FocusCR produces longer sentences with more information content and, at times, increasingly complex sentence structure.

Next, we observe that the average rating of the human-authored questions are surprisingly low across all categories, but particularly in Naturalness with a rating of 4.36. We attribute this to the crowd-sourcing methodology used to create the original SQuAD dataset. Nevertheless, we hypothesize that the average ratings of the gold questions will increase with larger sample sizes in subsequent human evaluation studies.

Inter-rater agreement was measured by comparing the Turkers' ratings to those of an expert annotator who is a native English speaking graduate student in Computational Linguistics. The expert annotator rated a random sample of 60 questions using a private version of the HIT created on Mechanical Turk. Then, the arithmetic mean of the three Turker ratings was calculated for each question and category of evaluation. The Pearson correlation coefficient between the expert annotator's rating and the means of the Turker ratings was $r=0.47$ for the Correct Information category, $r=0.38$ for the Answerability category, $r=0.20$ for Grammaticality, and $r=0.32$ for Naturalness. The significance of each correlation was calculated using a two-tailed test that resulted in $p<0.01$ for each category. We observe a positive correlation between the expert annotator and the Turker ratings in each category, although some of the the correlation strengths are less than ideal, particularly in the Grammaticality category. The consistent positive correlation across each category and their statistical significance provide evidence that the rating scheme is well defined, and that the Turkers are able to judge the quality of questions with relative reliability.

\begin{tabular}{lllll} 
Model & Grammar & Info. & Answer. & Natural \\
\hline Baseline & 4.23 & 3.78 & 3.73 & 4.10 \\
FocusCR & 4.20 & 4.13 & 4.13 & 4.09 \\
\hline Human & $\mathbf{4 . 4 0}$ & $\mathbf{4 . 4 0}$ & $\mathbf{4 . 4 7}$ & $\mathbf{4 . 3 6}$ \\
\hline
\end{tabular}

Table 9: Human Evaluation Results

\section{Related Work}

Much of the work on automatic question generation has been motivated by helping teachers in test creation (Mitkov and Ha, 2003; Heilman and Smith, 2010a; Labutov et al., 2015; Araki et al.,

\begin{tabular}{l|l}
\hline \# Model & \multicolumn{1}{|c}{ Sentences and Examples } \\
\hline S7 & $\begin{array}{l}\text { she publicly endorsed same sex marriage } \\
\text { on march 26, 2013, after the supreme court } \\
\text { debate on california 's proposition 8. }\end{array}$ \\
\hline $\begin{array}{l}\text { Q18 FocusCR } \\
\text { Q19 Focus }\end{array}$ & $\begin{array}{l}\text { what did beyonce publicly support? } \\
\text { what did madonna publicly endorsed on } \\
\text { march 26, 2013? }\end{array}$ \\
\hline \hline S8 & $\begin{array}{l}\text { west is one of the best-selling artists of all } \\
\text { time, having sold more than 32 million al- } \\
\text { bums and 100 million digital downloads } \\
\text { worldwide }\end{array}$ \\
\hline Q20 FocusCR & $\begin{array}{l}\text { how many grammy awards did he win? } \\
\text { how many grammy awards has madonna } \\
\text { Q21 Focus }\end{array}$ \\
\hline
\end{tabular}

Table 10: Additional Coreference Examples

2016; Chinkina and Meurers, 2017). Questions play an essential role in knowledge acquisition and assessment. It is standard practice for teachers to assess students' reading comprehension through question answering. Automatic question generation has the potential to assist teachers in the test creation process, thereby freeing teachers to spend more time on other aspects of the education process, and reducing the cost of receiving an education.

Automatic question generation has the potential to be useful in the areas of automatic Question Answering (QA) and Machine Comprehension of text. Recently, large datasets such as SQuAD (Rajpurkar et al., 2016), and MS MARCO (Nguyen et al., 2016) have facilitated advances in both areas. These datasets are expensive to create and consist of human authored (document, question, answer) triples with questions and answers either being collected from the web or created by crowd workers. Automatic Question Generation methods can be used to cheaply supplement resources available to QA models, further assisting in advancing QA capabilities. Indeed, Sachan and Xing (2018) have recently shown that a joint QA-QG model is able to achieve state-of-the art results on a variety of different QA related tasks.

Sequence-to-sequence Neural Network models have been shown to be effective at a variety of other NLP problems (Bahdanau et al., 2014; Rush et al., 2015; Juraska et al., 2018), and recent work has also applied them to QG (Du et al., 2017; Zhou et al., 2017; Yuan et al., 2017). As in other recent work on QG, we use an attentional Recurrent Neural Network encoder-decoder model that is similar to the model of Bahdanau et al. (2014). In this approach, the QG task is cast as a sequence-tosequence language modeling task. The input sen- 
tence, represented as a series of words, is mapped to an output series of words representing a question. Sequence-to-sequence models have several advantages over previous rule-based approaches to QG. First, they eliminate the need for large handcrafted rule sets - the model automatically learns how to perform the subtasks of Question Representation Construction and Question Realization. Another advantage is that the model does not rely on domain-specific keywords. In fact, in this approach the model is trained on examples from a variety of topics and then evaluated on examples from previously unseen topic domains.

Among the numerous approaches to question generation, our work is most similar to recent work applying neural network models to the task of generating short answer factoid questions.

Yuan et al. (2017) developed a Recurrent Neural Network (RNN) sequence-to-sequence model that generates questions from an input sentence. Their model is trained using supervised learning combined with reinforcement learning to maximize several auxiliary goals, including performance of a QA model on generated questions.

Du et al. (2017) present an attentional sequenceto-sequence model for question generation. Their model is similar to our baseline model but with one key difference: their model uses paragraphlevel information in addition to sentence-level information. They use an RNN encoder to embed the paragraph surrounding the sentence that contains the answer. Then the decoder's hidden state is initialized with the concatenation of the encoder's outputs and the paragraph embedding.

Sachan and Xing (2018) present an ensemble model that jointly learns both QA and QG tasks. The QG model is an RNN sequence-to-sequence model similar to that proposed by Du et al. (2017). First the QA and QG models are trained independent of each other on the labeled corpus. Then the QG model is used to create more questions from unlabeled data that are then answered by the QA model. A question selection oracle selects - based on several heuristics — a subsample of questions upon which to stochastically update each model. This process is repeated until both models cease to show improvement.

Heilman and Smith (2010a) present a system that generates fact-based questions similar to those in SQuAD using an "overgenerate-and-rank" strategy. Their system generates questions through use of hand crafted rules that operate on declarative sentences, transforming them into questions. In order to control quality, the output questions are filtered through a logistic regression model that ranks the questions on acceptability.

\section{Conclusion and Future Work}

We propose an encoder-decoder model for automatic generation of factual questions. We create a novel Neural Network architecture that uses two source sequence encoders; the first encoder being at the token level, and the second being at the sentence level. This enables the decoder to take into account word meaning and sentence meaning information while making decoding decisions. Also, the encoders are able to produce diverse encodings based on an answer focus feature. We demonstrate that this new model greatly improves on the state of the art in Question Generation when evaluated using automatic methods. We show that incorporating linguistic features into our model improves question generation performance as well. Lastly, a human evaluation confirms the improvement in quality of generated questions.

Currently, our system generates only factual questions for expository text. In future work we plan to explore question generation on other categories of text such as narrative discourse. One limitation of our system is that it relies on the existence of previously created answer phrases. Therefore, we would like to investigate methods of automatically extracting answer candidates from text, thus facilitating QG experiments on other categories of text that do not currently have large question-answer datasets.

\section{Acknowledgements}

This work was supported by NSF Cyberlearning EAGER grant IIS-1748056, NSF Robust Intelligence grant IIS-1302668-002, and an Amazon Alexa Prize 2017 Gift and 2018 Grant awarded to the Natural Language and Dialogue Systems Lab at UC Santa Cruz.

\section{References}

Manish Agarwal, Rakshit Shah, and Prashanth Mannem. 2011. Automatic question generation using discourse cues. In Proceedings of the 6th Workshop on Innovative Use of NLP for Building Educational Applications, page 19. Association for Computational Linguistics. 
Jun Araki, Dheeraj Rajagopal, Sreecharan Sankaranarayanan, Susan Holm, Yukari Yamakawa, and Teruko Mitamura. 2016. Generating questions and multiple-choice answers using semantic analysis of texts. In Proceedings of COLING 2016, the 26th International Conference on Computational Linguistics: Technical Papers, page 11251136.

Dzmitry Bahdanau, Kyunghyun Cho, and Yoshua Bengio. 2014. Neural machine translation by jointly learning to align and translate. arXiv:1409.0473 [cs, stat]. ArXiv: 1409.0473.

Satanjeev Banerjee and Alon Lavie. 2005. Meteor: An automatic metric for mt evaluation with improved correlation with human judgments. In Proceedings of the acl workshop on intrinsic and extrinsic evaluation measures for machine translation and/or summarization, pages 65-72.

Yllias Chali and Sadid A. Hasan. 2015. Towards topicto-question generation. Computational Linguistics, 41(1):120.

Maria Chinkina and Detmar Meurers. 2017. Question generation for language learning: From ensuring texts are read to supporting learning. In Proceedings of the 12th Workshop on Innovative Use of NLP for Building Educational Applications, page 334344.

Xinya Du, Junru Shao, and Claire Cardie. 2017. Learning to ask: Neural question generation for reading comprehension. arXiv:1705.00106 [cs]. ArXiv: 1705.00106 .

Jenny Rose Finkel, Trond Grenager, and Christopher D. Manning. 2005. Incorporating non-local information into information extraction systems by gibbs sampling. In Proc. of the 43nd Annual Meeting of the Association for Computational Linguistics (ACL 2005), pages 363-370.

Xavier Glorot and Yoshua Bengio. 2010. Understanding the difficulty of training deep feedforward neural networks. In Proceedings of the thirteenth international conference on artificial intelligence and statistics, page 249256.

Michael Heilman and Noah A. Smith. 2010a. Good question! statistical ranking for question generation. In Human Language Technologies: The 2010 Annual Conference of the North American Chapter of the Association for Computational Linguistics, HLT 10, page 609617. Association for Computational Linguistics.

Michael Heilman and Noah A. Smith. 2010b. Rating computer-generated questions with mechanical turk. In Proceedings of the NAACL HLT 2010 Workshop on Creating Speech and Language Data with Amazons Mechanical Turk, CSLDAMT 10, page 3540. Association for Computational Linguistics.

Sepp Hochreiter and Jürgen Schmidhuber. 1997. Long short-term memory. Neural computation, 9(8):1735-1780.
Juraj Juraska, Panagiotis Karagiannis, Kevin Bowden, and Marilyn Walker. 2018. A deep ensemble model with slot alignment for sequence-to-sequence natural language generation. In Proceedings of the 2018 Conference of the North American Chapter of the Association for Computational Linguistics: Human Language Technologies, Volume 1 (Long Papers), volume 1, pages 152-162.

Guillaume Klein, Yoon Kim, Yuntian Deng, Jean Senellart, and Alexander M. Rush. 2017. OpenNMT: Open-source toolkit for neural machine translation. In Proc. ACL.

Igor Labutov, Sumit Basu, and Lucy Vanderwende. 2015. Deep questions without deep understanding. In Proceedings of the 53rd Annual Meeting of the Association for Computational Linguistics and the 7 th International Joint Conference on Natural Language Processing (Volume 1: Long Papers), volume 1 , page 889898 .

Chin-Yew Lin. 2004. Rouge: A package for automatic evaluation of summaries. Text Summarization Branches Out.

Minh-Thang Luong, Hieu Pham, and Christopher D Manning. 2015. Effective approaches to attentionbased neural machine translation. arXiv preprint arXiv:1508.04025.

Christopher D Manning, Mihai Surdeanu, John Bauer, Jenny Rose Finkel, Steven Bethard, and David McClosky. 2014. The Stanford CoreNLP natural language processing toolkit. In ACL (System Demonstrations), pages 55-60.

Ruslan Mitkov and Le An Ha. 2003. Computer-aided generation of multiple-choice tests. In Proceedings of the HLT-NAACL 03 Workshop on Building Educational Applications Using Natural Language Processing - Volume 2, HLT-NAACL-EDUC 03, page 1722. Association for Computational Linguistics.

Jack Mostow and Wei Chen. 2009. Generating instruction automatically for the reading strategy of selfquestioning. In The 14th International Conference on Artificial Intelligence in Education, page 8.

Ramesh Nallapati, Bowen Zhou, Cicero Nogueira dos santos, Caglar Gulcehre, and Bing Xiang. 2016. Abstractive text summarization using sequence-tosequence rnns and beyond. arXiv:1602.06023 [cs]. ArXiv: 1602.06023.

Tri Nguyen, Mir Rosenberg, Xia Song, Jianfeng Gao, Saurabh Tiwary, Rangan Majumder, and Li Deng. 2016. Ms marco: A human generated machine reading comprehension dataset. arXiv:1611.09268 [cs]. ArXiv: 1611.09268.

Andrew M. Olney, Arthur C. Graesser, and Natalie K. Person. 2012. Question generation from concept maps. Dialogue \& Discourse, 3(2):7599. 
Kishore Papineni, Salim Roukos, Todd Ward, and WeiJing Zhu. 2002. Bleu: a method for automatic evaluation of machine translation. In Proceedings of the 40th annual meeting on association for computational linguistics, pages 311-318. Association for Computational Linguistics.

Jeffrey Pennington, Richard Socher, and Christopher Manning. 2014. Glove: Global vectors for word representation. In Proceedings of the 2014 conference on empirical methods in natural language processing (EMNLP), page 15321543.

Pranav Rajpurkar, Jian Zhang, Konstantin Lopyrev, and Percy Liang. 2016. Squad: 100,000+ questions for machine comprehension of text. arXiv:1606.05250 [cs]. ArXiv: 1606.05250.

Vasile Rus, Brendan Wyse, Paul Piwek, Mihai Lintean, Svetlana Stoyanchev, and Cristian Moldovan. 2010. The first question generation shared task evaluation challenge. Association for Computational Linguistics.

Alexander M Rush, Sumit Chopra, and Jason Weston. 2015. A neural attention model for abstractive sentence summarization. arXiv preprint arXiv:1509.00685.

Mrinmaya Sachan and Eric Xing. 2018. Self-training for jointly learning to ask and answer questions. Proceedings of the 2018 Conference of the North American Chapter of the Association for Computational Linguistics: Human Language Technologies, Volume 1 (Long Papers), 1:629640.

Abigail See, Peter J Liu, and Christopher D Manning. 2017. Get to the point: Summarization with pointergenerator networks. In Proceedings of the 55th Annual Meeting of the Association for Computational Linguistics (Volume 1: Long Papers), volume 1, pages 1073-1083.

Nitish Srivastava, Geoffrey Hinton, Alex Krizhevsky, Ilya Sutskever, and Ruslan Salakhutdinov. 2014. Dropout: a simple way to prevent neural networks from overfitting. The Journal of Machine Learning Research, 15(1):19291958.

Ilya Sutskever, Oriol Vinyals, and Quoc V Le. 2014. Sequence to sequence learning with neural networks. In Advances in neural information processing systems, pages 3104-3112.

Xingdi Yuan, Tong Wang, Caglar Gulcehre, Alessandro Sordoni, Philip Bachman, Saizheng Zhang, Sandeep Subramanian, and Adam Trischler. 2017. Machine comprehension by text-to-text neural question generation. In Proceedings of the 2 nd Workshop on Representation Learning for NLP, page 1525.

Qingyu Zhou, Nan Yang, Furu Wei, Chuanqi Tan, Hangbo Bao, and Ming Zhou. 2017. Neural question generation from text: A preliminary study. arXiv:1704.01792 [cs]. ArXiv: 1704.01792. 